\title{
Leseliste Top 5: Finanzierung in Krisenzeiten
}

In diesem Teil möchten wir Ihnen regelmäßig fünf besonders lesenswerte Bücher zu einem bestimmten Themengebiet vorstellen:

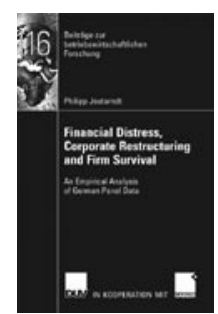

Jostarndt, Philipp:

Financial Distress,

Corporate

Restructuring and

Firm Survival

An Empirical Analysis of German Panel Data

Deutscher Universitätsverlag/Gabler Verlag, Wiesbaden 2007, 195 S., ISBN: 978-3-83500590-7, 55,90€

Im Rahmen seiner (englischsprachigen) Dissertation untersucht Jostarndt die Auswirkungen finanzieller Engpässe auf deutsche Unternehmen. Die empirische Untersuchung befasst sich mit den Folgen einer finanziellen Notlage für die Eigentümer- und Kontrollstrukturen, mit den Erfolgsfaktoren für Eigenkapitalerhöhungen und Fremdkapitalrestrukturierung in der Krise sowie mit der Entscheidung bezüglich gerichtlicher oder außergerichtlicher Restrukturierung.

Tipp: Dieses Buch wendet sich an Akademiker und interessierte Praktiker - insbesondere da es sich um einen der wenigen empirisch fundierten wissenschaftlichen Beiträge zur Thematik in Deutschland handelt.

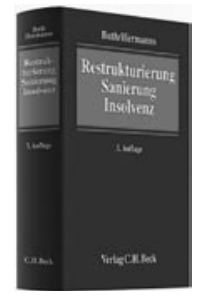

Buth, Andrea K./Hermanns, Michael (Hrsg.): Restrukturierung, Sanierung, Insolvenz

3. Auflage, C.H. Beck, München 2009, 874 S., ISBN: 978-3-406-57537-0, 92,00€

In der dritten Auflage erschienen, vermittelt das Buch einen aktuellen und umfassenden Zugang zur Problematik krisenbedrohter Unternehmen. Es wird weitgehend ein praxisorientierter Ansatz verfolgt, gestützt auf die Ergebnisse aktueller Studien sowie die Erkenntnisse aus verschiedenen Restrukturierungs- und Sanierungsprojekten. Die 40 Beiträge von Restrukturierungs- beratern, Rechtsanwälten, Finanzierungsexperten sowie Steuerberatern und Wirtschaftsprüfern beleuchten die Problematik zunächst aus den unterschiedlichsten Blickwinkeln und geben abschließend interessante Einblicke in sechs konkrete Fälle aus der Praxis.

Tipp: Das Buch wendet sich in erster Linie an Führungskräfte aus der Unternehmenspraxis. Dieser praxisnahe Ansatz kann jedoch auch für Wissenschaftler und Studenten viele interessante Anregungen liefern.

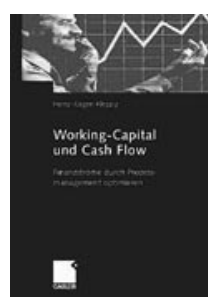

Klepzig, Heinz-Jürgen: Working-Capital und Cash Flow

Finanzströme durch

Prozessmanagement optimieren

Gabler Verlag, Wiesbaden 2008, 177 S., ISBN: 978-3834904232, 42,00€

In Krisenzeiten sind externe Finanzierungsquellen knapp und teuer. Klepzig befasst sich mit der Optimierung des internen Finanzierungspotenzials der Unternehmen. Nach einer kurzen aber umfassenden Darstellung des Begriffs Working-Capital und dessen Bedeutung für Prozessabläufe im Unternehmen beschreibt der Autor, wie prozessorientierte Anpassungen des Working-Capital-Managements erfolgen können. Insbesondere die Umsetzung entsprechender Veränderungen wird anhand verschiedener Projektkonzeptionen beispielhaft erläutert.

Tipp: Der Autor wendet sich in erster Linie an Praktiker, vor allem aus Finanzbereich und Unternehmensführung. Das Buch ist entsprechend umsetzungsorientiert gestaltet und gibt praktische Hilfestellungen.

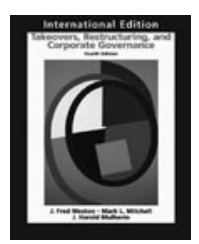

Weston, J. Fred/ Mitchell, Mark L./ Mulherin, J. Harold: Takeovers, Restructuring, and Corporate Governance

4. Auflage, Prentice Hall, Upper Sadlle River 2003, 720 S., ISBN: 978-0131225534, $84,99 €$
Dieses amerikanische Lehrbuch (in englischer Sprache) ist zunächst der Thematik Mergers \& Acquisitions gewidmet. In diesem Zusammenhang werden neben konzeptionellen und strategischen Fragen auch Bewertungsverfahren diskutiert. Zudem widmen die Autoren der Restrukturierung ein eigenes Kapitel. Dort werden Themen wie Desinvestitionsentscheidungen und finanzielle Restrukturierung behandelt. Die Inhalte werden immer wieder um empirische Informationen ergänzt.

TIPP: Das Buch ist vor allem für Studenten mit grundlegenden Kenntnissen der Unternehmensfinanzierung konzipiert. Durch die leicht verständliche Aufbereitung der Materie kann es auch für interessierte Wissenschaftler und Praktiker wertvolle Einsichten liefern. Für das Frühjahr 2010 ist die überarbeitete 5. Auflage angekündigt.

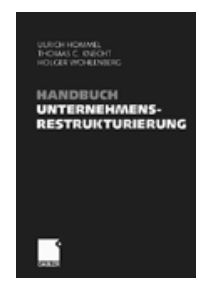

Hommel, Ulrich/Knecht, Thomas C./Wohlenberg, Holger (Hrsg.): Handbuch Unternehmensrestrukturierung

Gabler Verlag, Wiesbaden 2006, 1.489 S., ISBN 978-3-409-12654-0, 149,00€

Zusammengestellt aus 51 Beiträgen von namhaften Autoren aus Theorie und Praxis bietet das Buch auf fast 1.500 Seiten einen umfassenden Überblick über die Fragestellungen im Zusammenhang mit der Unternehmensrestrukturierung. Die inhaltlichen Schwerpunkte reichen von rechtlichen und ökonomischen Grundlagen und Sanierungskonzepten über Fragen der leistungswirtschaftlichen und finanziellen Sanierung bis hin zur Insolvenz als Option in der Restrukturierung. Dabei bleibt das Buch nicht nur an der Oberfläche, sondern liefert durchaus detaillierte Einsichten und Hintergründe zur Thematik.

TIPP: Das Buch wendet sich zunächst an Manager und Führungskräfte. Es bietet aber auch Wissenschaftlern und Studenten einen, durch verschiedene Fallbeispiele abgerundeten, Überblick über die Thematik.

Frank Bockisch und Dirk Hachmeister, Hohenheim 